\title{
Association of a Genetic Variant in ABCB1 Gene with the Tumor Size in Patients with Breast Cancer
}

DOI: $10.21859 / \mathrm{mci}$-supp-12

\author{
Soodabeh ShahidSales ${ }^{1}$, Davood Radmanesh ${ }^{2}$, Kazem Anvari ${ }^{1}$, \\ Saeideh Ahmadi-Simab ${ }^{1}$, Amir Avan ${ }^{3,4, *}$ \\ ${ }^{1}$ Cancer Research Center, Mashhad University of Medical Sciences, Mashhad, Iran \\ ${ }^{2}$ Department of Basic Medical Sciences, Neyshabur University of Medical Sciences, \\ Neyshabur, Iran \\ ${ }^{3}$ Department of Modern Sciences and Technologies, School of Medicine, Mashhad \\ University of Medical Sciences, Mashhad, Iran \\ ${ }^{4}$ Metabolic Syndrome Research Center, Mashhad University of Medical Sciences, \\ Mashhad, Iran
}

* Corresponding author: Amir Avan, Metabolic syndrome Research Center, Mashhad University of Medical Sciences, Mashhad, Iran.E-mail:avana@mums.ac.ir \& amir avan@yahoo.com

Keywords:
Breast Cancer
Prognostic Biomarker
Genetic Variant
CYP1B1
ABCB1

\section{Keywords}

Prognostic Biomarker

$\mathrm{ABCB} 1$

\begin{abstract}
Introduction Breast cancer is second leading cause of cancer-related-deaths in women. Therefore, identification of prognostic biomarkers, with the aim of spotting high-risk individuals along with proper management of patients during their treatment phase, is of paramount importance. It has been recently reported that genetic variants in ABCB1 and CYP1B1 genes correlates with many cancers including breast cancer, although their role as predisposition marker is still remained to be elucidated. The aim of current study was to evaluate the predictive value of two genetic variants, namely ABCB1/Rs2032582 and CYP1B1/Rs1056836 in breast cancer patients.

Materials and Methods: The present study was carried out on 112 breast cancer patients and 200 female individuals as the control group. Genomic DNA was extracted. Genotyping was carried out using TaqMan real time PCR method in case and control groups. The associations of this genetic variant were evaluated with breast cancer risk and pathological information of patients.

Results: Our data demonstrated that the genetic variant in $\mathrm{ABCB} 1$ gene was associated with tumor size, using recessive genetic model. In other words, patients with TT genotype were correlated with larger tumor size. Moreover, CYP1B1/Rs1056836 polymorphism did not have any correlation with metastasis status, nodal involvement, tumor size and stage of disease.

Conclusions: We demonstrated the association of $T \Gamma$ genotype in ABCB1/Rs2032582 with tumor size of breast cancer patients, indicating further studies in a larger population to show the value of emerging marker as a risk stratification biomarker in breast cancer.
\end{abstract}

